\title{
Advanced Operational Functionalities for a Low Voltage Microgrid Test Site
}

\author{
F. Adinolfi, Student, IEEE, F. D'Agostino, Student, IEEE, S. Massucco, Senior Member, IEEE, \\ M. Saviozzi, Student, IEEE, F. Silvestro, Member, IEEE
}

\begin{abstract}
This work aims to describe the implementation of a renewable energy based Microgrid test site facility at the University of Genova (Italy) and to depict the advanced functionalities there implemented within the national supported project Smartgen. The developed advanced Distribution Management System (DMS) is based on a Supervisory Control And Data Acquisition (SCADA) system for the remote monitoring of a Low Voltage Microgrid composed by a photovoltaic (PV) plant, an ion-lithium energy storage system, electrical load and a weather station. The implemented DMS is embedded with advanced functionalities which provide 36 hours forecasting of the load consumption and renewable generation. The forecasted data are also used to optimally program and manage the storage device. The proposed algorithms are described and also implementation aspects are reported within the paper.
\end{abstract}

Index Terms-Microgrid management, DMS, DER, storage system, load forecast, generation forecast, energy management

\section{INTRODUCTION}

The growing presence of the Distributed Energy Resources (DER) in the current distribution power systems at both Low (LV) and Medium Voltage (MV) levels together with customers participation to the liberalized energy market, are forcing Distribution System Operators (DSOs) to enhance actual Distribution Management Systems (DMSs). In particular, the moderns DMSs need to be equipped with several advanced functionalities to support the operators both during normal and extra-ordinary system operation [1]. According to [2] a Microgrid can be defined as the portion of the distribution system connected downstream of the MV/LV substation where DERs, which include both Distributed Generation (DG) and storage units, are located together with different end user types.

The integration of DER and the development of public/private Microgrids have been addressed by a number of publications in literature. In particular, in [3] authors focused on photovoltaic (PV) integration with storage equipment by proposing an optimized energy management system; while in [4] a rural Microgrid with presence of different renewables and conventional resources is operated according with demand side management techniques.

F. Adinolfi, F. D'Agostino, S. Massucco, M. Saviozzi and F. Silvestro are with DITEN (Electrical, Electronic, Telecommunication Engineering and Naval Architecture Department), University of Genova, via Opera Pia 11a, 16145 Genova, Italy (email: \{francesco.adinolfi; stefano.massucco; federico.silvestro\} @unige.it and \{fabio.dagostino; matteo.saviozzi\} @edu.unige.it,)
This work has been developed within the project "Smartgen", supported by the Italian Ministry of Economic Development. Smartgen develops a novel DMS for MV and LV operators embedded with advanced functionalities studied to meet the new needs in the actual scenario [5]. Three different pilot sites have been identified and equipped during the project to test and validate the different functionalities proposed. One of these sites is the Virtual Power Plant (VPP) of the University of Genova (Italy), which includes $15 \mathrm{MV} / \mathrm{LV}$ substations where the different Faculties are located. Each substation consumption is remotely monitored and the energy consumption metering is acquired with 1 minute sampling by the DMS. Moreover, the test site includes a Microgrid installed at the Faculty of Economics. The actual version of the proposed DMS is running continuously since April 2014. The current paper is so organized: in Section II the Microgrid site is presented, while in Section III the mathematical aspects of the proposed algorithms are provided. In Section IV field implementation and test results are showed. Finally some short conclusions are presented in Section V.

\section{TEST Site MicRogrid}

Within the project "Smartgen" the University of Genova has realized a LV Microgrid located in the Faculty of Economics and depicted in Figure 1.

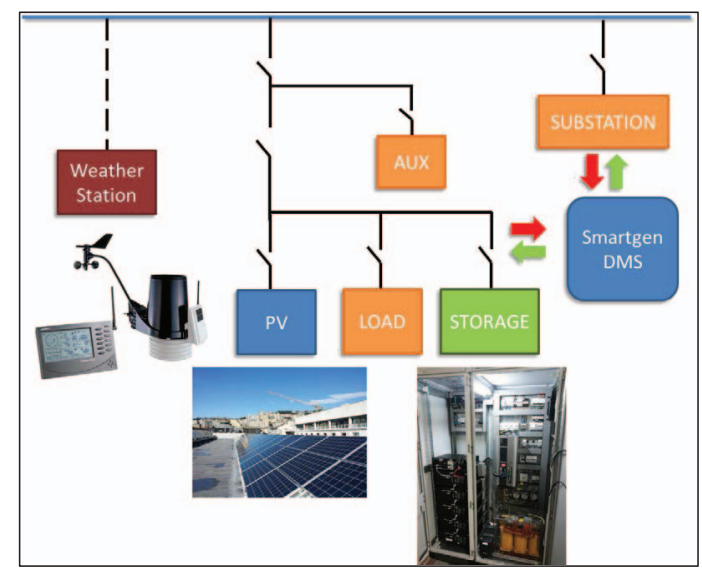

Figure 1. The Low Voltage Microgrid test site at University of Genova 
The system is composed by: $20 \mathrm{kWp}$ of photovoltaic panels installed on the building roof and connected to the AC busbar through two $10 \mathrm{~kW}$ inverters; a $10 \mathrm{~kW}-12 \mathrm{kWh}$ ion-lithium storage system located at ground level, in the local MV/LV substation, with the auxiliary services loads; and finally the local monitored load which collects the energy consumption of the building. On the roof a weather station is located which provides a number of meteorological measures necessary for the forecast algorithms. All the mentioned devices communicate with the DMS through specific converters that allow Modbus communication protocol via TCP/IP with a sampling rate of 0.1 seconds.

\section{ADVANCED OPERATIONAL FUNCIONALITIES}

The above illustrated Microgrid has been used to validate three different advanced functionalities, implemented in the Smartgen DMS: the forecast of renewables generation (AGF); load consumption (ALF) and the optimal programming (APO) of DER, such as the available lithiumion storage system. The algorithms proposed are illustrated in details. All output data both forecasted and set points commands have 15 minutes granularity. All the weather forecasting data belong to a public weather provider available online at the website.

\section{A. Algortithm for Generation Forecast (AGF)}

The implemented renewables generation forecast functionality is based on a hybrid approach, which combines both deterministic and linear regression techniques. In particular the PV resource has been considered being it available in the test site. The procedure received four different data types in input as showed in Figure 2.

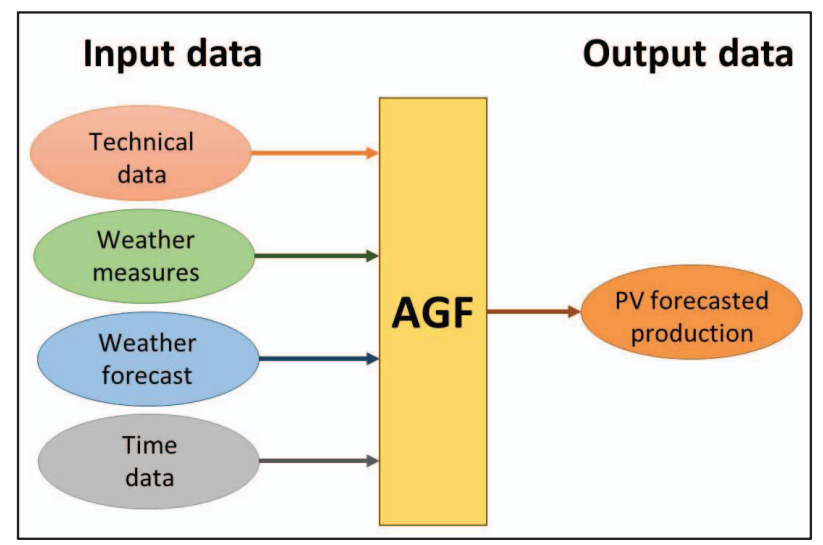

Figure 2. Algorithm for Generation Forecast (AGF) architecture

The technical data consist in: latitude, longitude, PV panels efficiency (eff), tilt angle (tilde), optimal operational temperature (noct), installed capacity $\left(P_{\text {peak }}\right)$; while the weather measures used are temperature $\left(\right.$ temp $\left._{\text {mea }}\right)$ values. The weather forecast information are the sky clearness (sky, $0=$ clear, $100=$ dark sky), rainfall probability (pop), temperature $\left(\right.$ temp $\left._{\text {forecasted }}\right)$ and humidity $\left(\right.$ hum $\left._{\text {forecasted }}\right)$. Finally
AGF receives also time data inputs about day of the year and time of the day.

Once collected all the inputs requested the AGF procedure is composed by 8 steps:

1. Forecast of solar irradiation in clear-sky situation $\left(\mathrm{rad}_{\text {clear }}\right)$ based on the site geographical coordinates and time data.

2. Panels tilt angle impact $\left(\right.$ tilde $\left._{\text {effect }}\right)$.

$$
\text { tilde }_{\text {effect }}=\sin (\text { tilde }+\alpha)
$$

where $\alpha$ is the solar high angle.

3. Calculation of solar irradiation $\left(\mathrm{rad}_{\text {temp } / \text { hum }}\right)$ affected by the forecasted temperature and humidity respectively temp $p_{\text {effect }}$ and hum $_{\text {effect }}$.

$$
\mathrm{rad}_{\text {temp/hum }}=\operatorname{rad}_{\text {clear }} \cdot \text { hum }_{\text {effect }} \cdot \text { temp }_{\text {effect }}
$$

4. Calculation of expected production affected by tilt angle, temperature and humidity forecasted ( $\left.P_{\text {temp } / \text { hum_tilde }}\right)$.

5. Calculation of the Nominal Operating Cell Temperature effect - NOCT $\left(N O C T_{\text {effect }}\right)$, which decreases the panel performance comparing with rated temperature one.

$N O C T_{\text {effect }}=\frac{(N O C T-20) \mathrm{rad}_{\text {temp } / \text { hum }}}{G_{0}}+$ temp $_{\text {mea } / \text { forecasted }}$

where $G_{0}=1000 \mathrm{~W} / \mathrm{m}^{2}$ is the irradiation standard value.

6. Power production estimation considering inverters and DC losses impact rend std $_{(}\left(P_{s t d}\right)$.

$$
P_{\text {std }}=\text { rend }_{\text {std }} \cdot P_{\text {temp } / \text { hum_tilde }}
$$

7. Calculation of sky clearness index impact on PV generation $\left(P_{\text {sky }}\right)$.

$$
P_{s k y}=P_{s t d} \cdot\left(\frac{100-k(s k y) \cdot s k y}{100}\right)
$$

where $k(s k y)$ is the parameter which counts the sky index impact. It has been evaluated adopting linear regression technique based on historical plant production data.

8. Final evaluation of the expected production introducing the sky-covering index $\left(P_{p o p}\right)$.

$$
P_{p o p}=P_{s k y} \cdot\left(\frac{100-k(p o p) \cdot p o p}{100}\right)
$$

where $k$ (pop) is the parameter which considers the index pop (rainfall probability). Similarly to the previous case it changes according to the plant characteristic and it has been evaluated by analyzing the historical production database.

The final forecasted power production is equal to what obtained at step 8:

$$
P_{\text {tot }}=P_{\text {pop }}
$$

\section{B. Algortithm for Load Forecast (ALF)}

The Algorithm for Load Forecasting (ALF) is based on an Artificial Neural Network (ANN) architecture and provides the expected power absorption for the next 36 hours [6]. 
The methods based on ANN are composed of three different phases:

1. Training: a large database including historical consumption data is used to train the network to identify the correlations between inputs and known output.

2. Test: the ANN does not receive the output values anymore, so the forecast provided by the algorithm is compared with the real measured output.

3. Online operation: if ANN passed the test phase the algorithm is implemented in the DMS.

The ANN architecture here proposed for the day-ahead forecasting has a multi-layer perceptron structure. This particular type of ANN consists of three parts: input layer, hidden layer, and output layer:

- the input layer catches requested data;

- the hidden layer is not interfaced with the outside world;

- the output layer provides the ANN output to the outside world.

The proposed ALF uses only one hidden layer. This choice allows to achieve good results in terms of computational speed during the training phase and of ANN architecture simplicity. As depicted in Figure 3, different type of data have been used as input. In particular the ANN inputs used to forecast the load are the 144 values (15 minutes granularity for the considered set of 36 hours) reported below:

- average load consumption of the previous 36 hours;

- 144 values which represent the 36 h-ahead load;

- 144 values related to the 7 days-ahead load;

- 144 values related to the 14 days-ahead load;

- 144 values representing the 36 hours ahead temperature.

The transfer function used in the hidden layer and the output layer has been the Hyperbolic Tangent Sigmoid Transfer Function [7]:

$$
y=\operatorname{tansig}(x)=\frac{2}{\left[\left(1+e^{-2 x}\right)-1\right]}
$$

This function is characterized by the fact that its slope must approach zero as the input gets larger. This causes a problem when typical steepest descent methods are used to train a multilayer ANN with sigmoid functions, because the gradient can has a very small magnitude and, therefore, causes small changes in the weights, even though the weights are far from their optimal values. The purpose of the Resilient backPROPagation (RPROP) [8] training algorithm is to eliminate these harmful effects of the magnitudes of the partial derivatives. Only the sign of the derivative can determine the direction of the weight update; the magnitude of the derivative has no effect on the weight update.

Once have been defined ANN type, transfer functions and training algorithm some preliminary tests have been carried out to identify the number of neurons to adopt. The results highlight that 15 neurons provide good performances. The final ANN architecture is summarized in Figure 3.

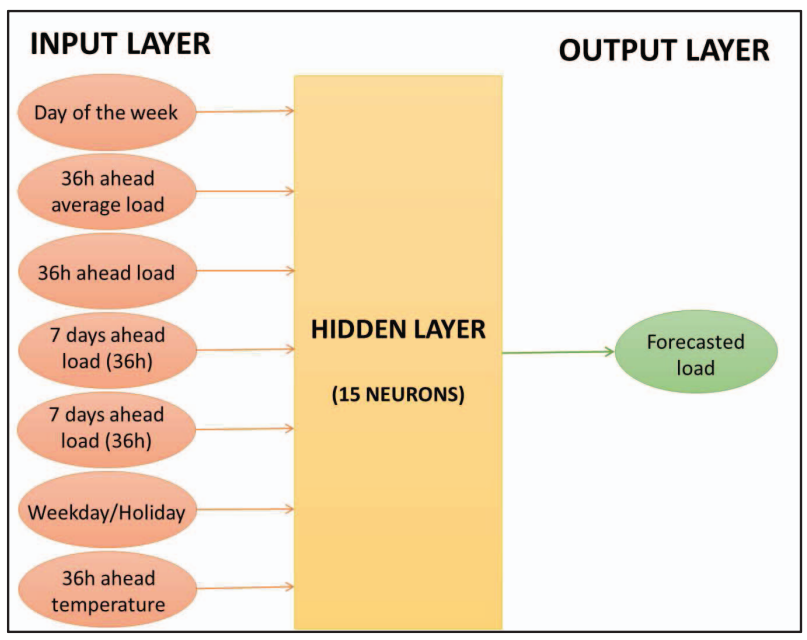

Figure 3. ANN architecture for the Load Forecast Algorithm (ALF)

\section{Algorithm for working-Point Optimization (APO)}

To optimally define the working point of DERs (generators, storage devices and controllable loads), the DMS has been equipped with the Algorithm for working-Point Optimization (APO) here illustrated.

The APO functionality is based on an economic scheduler which provides the optimal set-point profile for each dispatchable device installed in the Microgrid, in terms of the required energy. The outputs of APO consist of 96 values, one every 15 minutes on a 24 hours horizon. These values which represent the active power set point of each dispatchable resource available. The mathematical formulation of the optimization procedure can be expressed as:

$$
\min _{P^{r}}\left(\sum_{r=1}^{R} \sum_{j=1}^{N} c_{j, r} \Delta t P_{j}^{r}\right),
$$

where the horizon time is sub-divided into $R$ intervals $(\Delta t), N$ is the number of dispatchable devices, $P^{r}=\left(P_{1}^{r}, \ldots, P_{N}^{r}\right)$ is the active power vector of the $N$ units at instant $r$, and $c_{j, r}$ is the energy cost per kWh of the $j$-th unit at the time $r$. This implies that the product between $c_{j, r}$ and $\Delta t$ is the final cost, i.e. the optimization object.

According to what illustrated in Figure 4, APO receives inputs from different sources. In particular the algorithm needs to know the site characteristics: the technical constrains of the dispatchable resources, the State of Charge (SoC) of storage devices available and the energy costs of the local electric energy market [9],[10]. Moreover to optimally schedule the controllable resources forecasted data about renewables production and loads absorption are requested. These data are provided by AGF and ALF respectively whereas weather information are collected by the weather station installed onsite and website provider. 


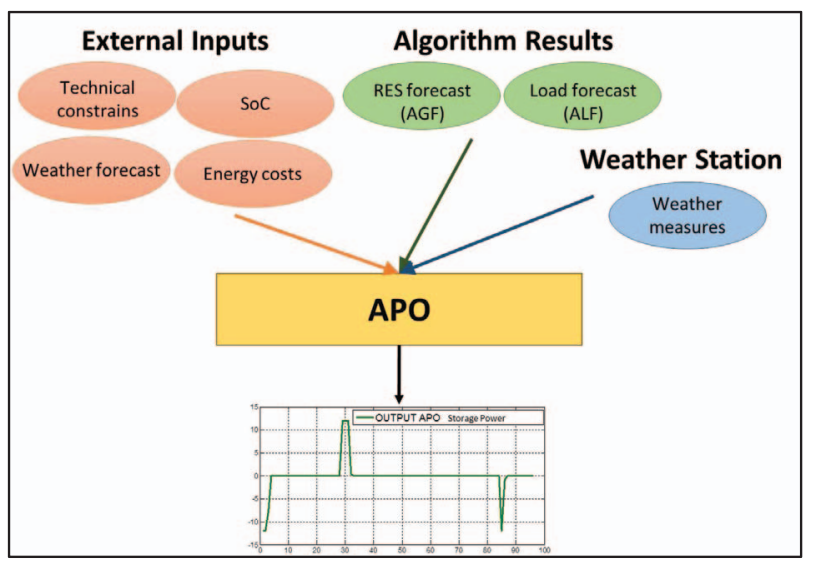

Figure 4. Algorithm for working-Point Optimization (APO) architecture

If $S$ are the production/consumption units and $L$ are the storage units, the indices vector $h=\{1, \ldots, N\}=$ $\{1, \ldots, S, S+1,, \ldots, S+L, S+L+1, \ldots N\}$ can be defined. Then $j \in h$ represents a system unit. The global amount of not-dispatchable units is equal to $N P$ and their power production is denoted by $P_{n p 1}, \ldots, P_{n p N P}$, while $E_{j}^{r}$ is the energy stored at time $r$ into the $j$-th unit. Then the technical constrains can be defined as follows:

- electrical load balance $P_{\text {load }}$

$$
\sum_{j=1}^{N} P_{j}^{r}=P_{\text {load }_{e}}^{r}-\sum_{k \in N P} P_{n p_{k}}^{r}, \quad r=1, \ldots, R ;
$$

- integral constrains for storage units

$$
\begin{gathered}
E_{j}^{r}=E_{j}^{r-1}-\Delta t P_{j}^{r-1}, \\
E_{j}^{R+1}=E_{j}^{R}-\Delta t P_{j}^{R}, \quad E_{j}^{1}=E_{j}^{0} \\
r=2, \ldots, R, \quad j=S+1, \ldots S+L,
\end{gathered}
$$

- maximum/minimum power operational constrains and energy limit

$$
\begin{gathered}
P_{j}^{\min } \leq P_{j}^{r} \leq P_{j}^{\max }, \quad \forall r, j, \\
E_{j} \leq E_{j}^{\max } j=S+1, \ldots, S+L
\end{gathered}
$$

Moreover the energy efficiency of the storage units is taken into account considering:

$$
P_{c h}=k_{c h}(T, i) P_{\text {in }} \quad P_{d c h}=k_{d c h}(T, i) P_{\text {out }}
$$

where $P_{c h}$ and $P_{d c h}$ are referring to charge/discharge power, $k_{c h}$ and $k_{d c h}$ are the storage efficiencies which are function of temperature $(T)$ and current $(i)$. The so defined optimization problem is solved adopting the Simplex method of a linear programming solution.

\section{FIELD IMPLEMENTATION AND TEST RESUlTS}

As illustrated within Section III the forecasting algorithms proposed request to be tested or trained before being put online. In particular, to evaluate the AGF performance, the results collected during one month test have been analyzed adopting the normalized Root Mean Square Error (nRMSE) performance index, defined as:

$$
n R M S E=\frac{\sqrt{\frac{1}{N} \sum_{t=1}^{N}\left(Y_{t}-F_{t}\right)^{2}}}{\sqrt{\frac{1}{N} \sum_{t=1}^{N}\left(Y_{t}\right)^{2}}}
$$

where $N$ is the number of forecasted values, $Y_{t}$ is the actual value of generated power within a 15 minutes period $t$ and $F_{t}$ is the forecasted value in the same period $t$. The nRMSE provides information about the mean percentage error. The numerical result obtained during the test of AGF performed from May $15^{\text {th }}$ to June $15^{\text {th }}$ is nRMSE $=0.29[\mathrm{pu}]$. A graphic evaluation is available observing the plots depicted in Figure 5 , which represent May $15^{\text {th }}$ and June $11^{\text {th }}$ tests respectively.
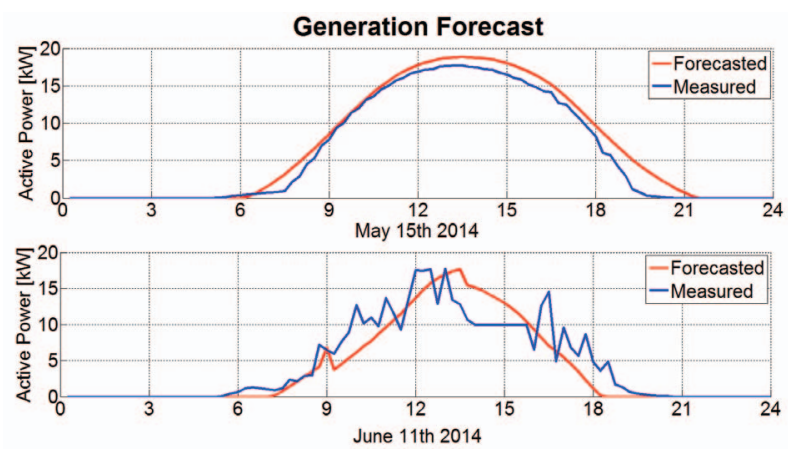

Figure 5. AGF results and measured PV production (May $15^{\text {th }} 2014$ and June $11^{\text {th }} 2014$ )

The results obtained show the effectiveness of the proposed method: the nRMSE index is below the $30 \%$ threshold. It has been considered enough accurate and satisfying to implement AGF on the operating DMS.

Concerning the load forecast function, ALF, the results of the training phase, described in Section III.B, have been analyzed by using the performance indices MAPE (Mean Absolute Percentage Error) and Daily Peak MAPE, defined as:

$$
\begin{gathered}
\text { MAPE }=\frac{1}{N} \sum_{t=1}^{N} \frac{\left|Y_{t}-F_{t}\right|}{Y_{t}} \cdot 100 \%, \\
\text { Daily Peak MAPE }=\frac{\left|\max \left(Y_{t}\right)-\max \left(F_{t}\right)\right|}{\max \left(Y_{t}\right)} \cdot 100
\end{gathered}
$$

Whereas the MAPE index provides an estimation of the global percentage error by the algorithm, the Daily Peak MAPE is useful to analyze the error in presence of the maximum load consumption during the day. The ANN training phase has been evaluated by a test set indicated in TABLE I. The results achieved are collected in TABLE I.

TABLE I. Neural Network training phase for the ALF function

\begin{tabular}{|c|c|c|c|}
\hline \multicolumn{2}{|c|}{ Data Set Period } & \multicolumn{2}{c|}{ Performance indices } \\
\hline Training Set & Test Set & MAPE & Daily Peak MAPE \\
\hline $01 / 01 / 2014-$ & $02 / 25 / 2014-$ & $11.23 \%$ & $8.62 \%$ \\
$02 / 24 / 2014$ & $03 / 10 / 2014$ & & \\
\hline
\end{tabular}


Both performance indices showed that the training phase could be considered completed. Thus the ALF functionality has been embedded in the DMS.

For the APO implementation knowledge of storage system efficiency is needed. The constant current discharging tests performed on the field have provided the performance curve reported in Figure 6.

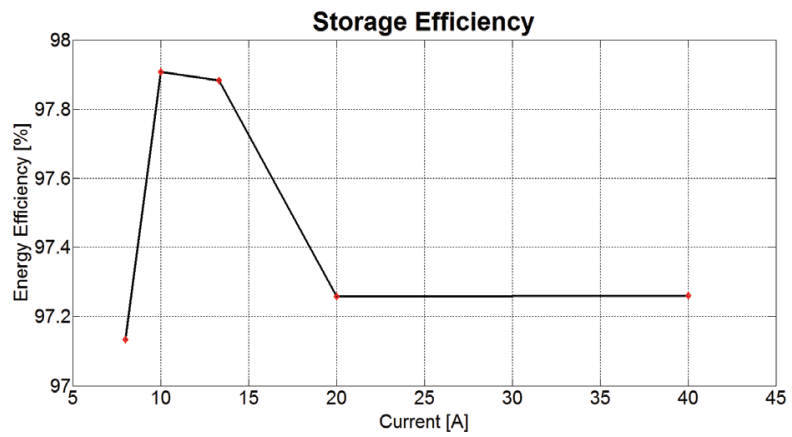

Figure 6: Energy efficiency for different discharge currents

Finally the two AGF and ALF algorithms have been combined as input for the APO optimal scheduling functionality. In Figure 7 the ALF and AGF forecasted data are depicted in the above part while the two subplots below represent the active power and the SOC set points.
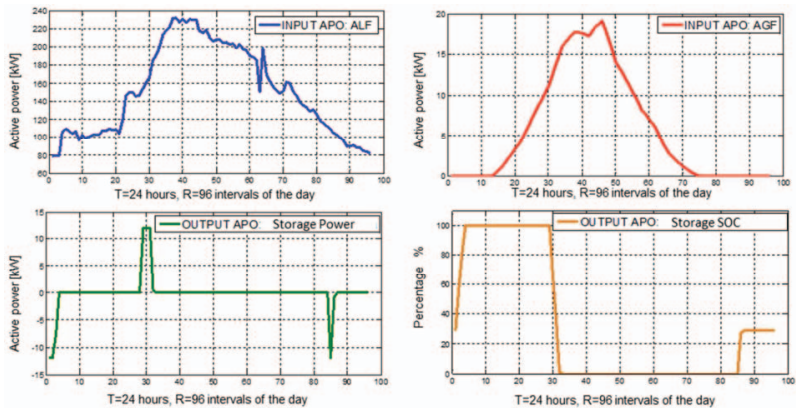

Figure 7. APO operation: ALF (blue) and AGF (red) inputs, active power (green) and SoC (yellow) set point outputs.

Once completed the training and test phases of the algorithms, all the three proposed functionalities have been implemented on the operating DMS, interfaced to the field via SCADA system. with the field, and currently operating. Figure 8 shows the Human Machine Interface (HMI) of the running DMS: at the top, in central position, the green buttons represent the commands used by the operator to initiate a function. A left click on one button starts the procedure to obtain the corresponding algorithm results. Moreover ALF, AGF and APO have been scheduled to be executed every day to obtain the forecasted consumption, production and set points for the next day. The outputs of each functionality are stored in dedicated a database. Furthermore a graphical representation is available (see Figure 8) and can be opened by the operator for launching individually each of the developed functions (AGF, ALF, APO).

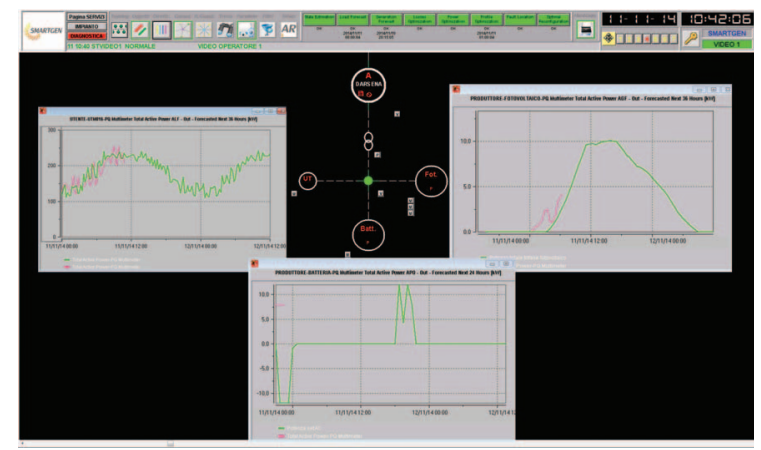

Figure 8. "Smartgen" DMS layout, graphical results of AGF, ALF and APO functionalities.

\section{CONCLUSIONS}

This work illustrates an operating example of field implementation of some advanced functionalities in a modern DMS. The forecast of load consumption and renewables production, which are two of the most discussed issues in the current scenario, have been faced through the proposition of tested algorithms based on Neural Network and deterministic approaches. Moreover these functionalities have been combined to achieve the remote optimal programming of a storage device used to realize a smart Microgrid site.

\section{REFERENCES}

[1] Meliopoulos, A.P.S.; Polymeneas, E.; Zhenyu Tan; Renke Huang; Dongbo Zhao, "Advanced Distribution Management System," Smart Grid, IEEE Transactions on , vol.4, no.4, pp.2109-2117, Dec. 2013

[2] Katiraei, F.; Iravani, R.; Hatziargyriou, N.; Dimeas, A., "Microgrids management", Power and Energy Magazine, IEEE, vol.6, no.3, pp.5465, May-June 2008

[3] Chen, C.; Duan, S.; Cai, T.; Liu, B.; Hu, G., "Smart energy management system for optimal microgrid economic operation", Renewable Power Generation, IET, vol.5, no.3, pp.258267, May 2011

[4] Palma-Behnke, R.; Benavides, C.; Lanas, F.; Severino, B.; Reyes, L.; Llanos, J.; Saez, D., "A Microgrid Energy Management System Based on the Rolling Horizon Strategy", Smart Grid, IEEE Transactions on, vol.4, no.2, pp.996-1006, June 2013

[5] S. Bianchi, A. Borghetti, S. Massucco, F. Napolitano, C.A. Nucci, M. Pentolini, G. Petretto, S. Scalari, F. Silvestro, G. Troglio, G. Viano, "Development and Validation of Innovative Methods and Tools for the Management of Active Distribution Networks: the SmartGen project", Medpower 2014, Athens 2-5 November 2014

[6] A. Bagnasco, M. Saviozzi, F. Silvestro, A. Vinci, S. Grillo, E. Zennaro, "Artificial Neural Network Application to Load Forecasting in a Large Hospital", 13th International Conference on Probabilistic Methods Applied to Power Systems (PMAPS), Durham, 7-10 July

[7] Matlab Server Online Manual, The Mathworks Inc.

[8] J. Nocedal, S. J. Wright, Numerical Optimization, Springer, 1999.

[9] F. Adinolfi, G. M. Burt, P. Crolla, F. D’Agostino, M. Saviozzi, F. Silvestro, "Distributed Energy Resources Management in a Low Voltage Test Facility", accepted for publication on IEEE Transactions on Industrial Electronics, 2014.

[10]Borghetti, A.; Bosetti, M.; Grillo, S.; Massucco, S.; Nucci, C.A.; Paolone, M.; Silvestro, F., "Short-Term Scheduling and Control of Active Distribution Systems With High Penetration of Renewable Resources," Systems Journal, IEEE, vol.4, no.3, pp.313-322, Sept. 2010 . 